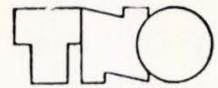

COMMUNICATION NR. 168

OF THE

CENTRAL LABORATORY

CORRECTIONS FOR SHEAR AND ROTATORY INERTIA ON FLEXURAL VIBRATIONS OF BEAMS

by

C. J. NEDERVEEN

and

F. R. SCHWARZL 
BRIT. J. APPL. PHYS., 1964, VOL. 15

\title{
Corrections for shear and rotatory inertia on flexural vibrations of beams
}

\author{
C. J. NEDERVEEN and F. R. SCHWARZL \\ Central Laboratory T.N.O., Delft, The Netherlands \\ MS. received 22nd October 1963
}

\begin{abstract}
Different correction formulae for the influence of shear and rotatory inertia on flexural vibrations of freely supported beams are compared with the exact solution. It appears that in most cases a simple formula is sufficient because of the appearance of a constant which is not accurately known, viz. the shear deflection coefficient.
\end{abstract}

One method for the determination of Young's modulus consists in subjecting a beam of the material to bending vibrations. The modulus is calculated from the resonance frequency, when the mass and dimensions of the specimen are given. For beams which are thick compared with the wavelength shear and rotatory inertia must be taken into account. In practice these effects are accounted for by the application of corrections according to a simple theory. In these correction formulae an empirical constant appears, viz. the shear deflection coefficient $s$ which accounts for the non-uniform distribution of shear stresses over the cross section. The value of this coefficient is not accurately known. Because of this uncertainty the region of applicability of the correction formulae is restricted, as will be shown.

In simple theory the lateral dimensions of the beam are assumed to be small with respect to wavelength. Then the vibrations are governed by (Rayleigh 1945)

$$
\frac{i^{2} E}{\rho} \frac{\partial^{4} y}{\partial x^{4}}+\frac{\partial^{2} y}{\partial t^{2}}=0
$$

where $i=$ radius of gyration of cross section, $E=$ Young's modulus, $\rho=$ density, $y=$ lateral deflection, $x=$ distance along the beam and $t=$ time. Solutions of equation (1) are of the form

$$
y=\cos \omega t \exp \left[ \pm( \pm 1)^{1 / 2} \frac{m x}{l}\right]
$$

where $\omega$ is the angular frequency and $l$ the beam length; $m$, positive and real, is fixed by

$$
E_{0}=\frac{\omega^{2} l^{4} \rho}{m^{4} i^{2}}
$$

where index 0 indicates 'according to simple theory'. The boundary conditions for a beam free at $x=0$ and $x=l$ are $\partial^{2} y / \partial x^{2}=0$ and $\partial^{3} y / \partial x^{3}=0$. Substitution in a linear combination of the four solutions (2) gives as a condition for $m$ :

$$
\cos m \cosh m=1 ;
$$

to a good approximation this yields

$$
\begin{aligned}
& m_{1}=1 \cdot 5056 \pi \\
& m_{p}=\frac{1}{2}(2 p+1) \pi \quad p>1 .
\end{aligned}
$$


The complete differential equation including shear and rotatory inertia is (Timoshenko 1921, 1953)

$$
\frac{E i^{2}}{\rho} \frac{\partial^{4} y}{\partial x^{4}}-i^{2}\left(1+s \frac{E}{G}\right) \frac{\partial^{4} y}{\partial x^{2} \partial t^{2}}+\frac{\partial^{2} y}{\partial t^{2}}+\frac{\rho s i^{2}}{G} \frac{\partial^{4} y}{\partial t^{4}}=0
$$

where $G$ denotes the shear modulus and $s$ is the shear deflection coefficient. An exact solution for the boundary conditions mentioned was carried out by Goens (1931) and was found to be $k$, the solution of the transcendental equation:

$$
\begin{gathered}
\left(\frac{\tan \frac{1}{2} \beta k}{\tanh \frac{1}{2} \alpha k}+\frac{\beta \eta}{\alpha \epsilon}\right)\left(\frac{\tan \frac{1}{2} \beta k}{\tanh \frac{1}{2} \alpha k}-\frac{\alpha \epsilon}{\beta \eta}\right)=0 \\
\alpha, \beta=\left[\left(B^{2} k^{4}+1\right)^{1 / 2} \pm A k^{2}\right]^{1 / 2} \\
\epsilon, \eta=\left(B^{2} k^{4}+1\right)^{1 / 2} \pm B k^{2} \\
A, B=\frac{1}{2} \frac{i^{2}}{l^{2}}\left(s \frac{E}{G} \pm 1\right) .
\end{gathered}
$$

Modulus $E$ then follows from

$$
E=\frac{\omega^{2} l^{4} \rho}{k^{4} i^{2}}=\frac{m^{4}}{k^{4}} E_{0} .
$$

For practical calculations a very good approximation for Young's modulus $E_{\mathbf{G}}$ was given by Goens (1931):

$$
E_{\mathrm{G}}=E_{0}\left[1+2 A m^{2} \phi^{2}+4(A-2 B) m \phi-\frac{\left(A^{2}-B^{2}\right) m^{4}}{1+2 A m^{2}}\right]
$$

where $\phi=0.9825$

$$
\begin{aligned}
& \phi=1 \cdot 0008 \quad p=2 \\
& \phi=0.99997 \quad p=3 \\
& \phi=1 \cdot 0000 \quad p \geq 4 \text {. }
\end{aligned}
$$

A simple approximation is found when, following Timoshenko $(1921,1953)$, the function $y=\cos \omega t \exp ( \pm j m x / l)$ is substituted in equation (6). If we neglect $\rho s i^{2} \omega^{2} / G$ with respect to unity and consider $E / G$ as constant, the resulting equation can be solved for $E_{\mathrm{T}}$ :

$$
E_{\mathrm{T}}=E_{0}\left[1+\frac{i^{2}}{l^{2}}\left(1+s \frac{E}{G}\right) m^{2}\right]=E_{0}\left(1+2 A m^{2}\right) .
$$

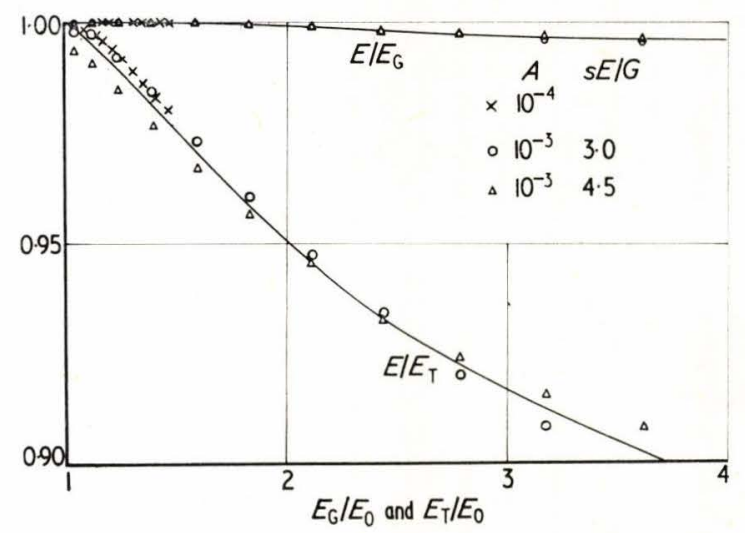

Figure 1. Exactness of the corrections as a function of their magnitudes.

$E=$ exact solution, $E_{\mathrm{G}}=$ Goens' approximation, $E_{\mathrm{T}}=$ Timoshenko's approximation, $E_{0}=$ approximation neglecting shear and rotatory inertia. 
For comparison the two corrections and the exact solution have been calculated, using a ZEBRA computer, up to $p=15$ for two values of $A$ and two values of $s E / G$. The results are shown in figure 1 , where the reciprocal relative deviations of the modulus with respect to the exact modulus are plotted against the magnitude of the correction terms of equations (9) and (10). As may be seen from the diagram, the approximation of Goens agrees with the exact solution to within $1 \%$ for corrections up to a factor of 4 . The approximation of Timoshenko already gives deviations of $1 \%$ with a correction of $20 \%$. These deviations may be compared with deviations in the result due to the uncertainty in the value of $s$. For wood, measurements by Hearmon (1958) resulted in $s=1 \cdot 06 \pm 0 \cdot 05$. Different theories have led to a great variety of calculated $s$ values, from 1.0 up to 1.5 depending on line of attack, type of boundary conditions, shape of cross section, Poisson's ratio and wavelength (Hearmon 1958). For the case considered here, viz. a rectangular beam with both ends free, the most probable value of $s$ seems to be 1.15 with an uncertainty of about $10 \%$. The influence on Young's modulus of a variation of $-10 \%$ in $s$ is given in figure 2 as a function of the magnitude of the correction.

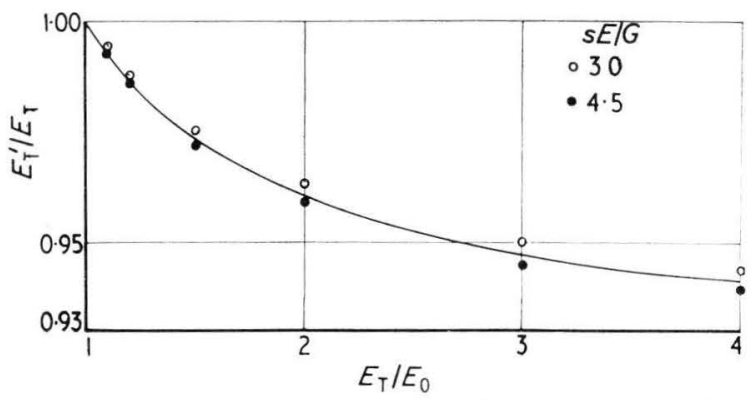

Figure 2. Deviation of the correction as a function of its magnitude for a $10 \%$ decrease in $s$. $E_{\mathrm{T}}=$ Timoshenko's approximation, $E_{\mathrm{T}^{\prime}}=$ Timoshenko's approximation with a $10 \%$ decrease in $s$, $E_{0}=$ approximation neglecting shear and rotatory inertia.

Comparing figures 1 and 2 we conclude that the deviations from the exact value of Timoshenko's approximation are about as large as the deviations caused by inexact knowledge of the shear deflection coefficient. Thus there is obviously no reason to use a more intricate formula than the simple one proposed by Timoshenko.

We express our thanks to Dr. W. H. Muller of the Statistics Department T.N.O. for his valuable help in solving the equations.

\section{References}

Goens, E., 1931, Ann. Phys., Lpz., 11, 649.

Hearmon, R. F. S., 1958, Brit. J. Appl. Phys., 9, 381.

RaYleigh, J. W. S., 1945, The Theory of Sound, Vol. 1 (New York: Dover), p. 260.

Timoshenko, S. P., 1921, Phil. Mag., 41, 744.

1953, Collected Papers (New York: McGraw-Hill), p. 288. 